J3eA - Vol. 1 - 1 (2002).

DOI : 10.1051/bib-j3ea:2002001

\title{
Instrumentation virtuelle sur le World Wide Web pour faire des mesures réelles
}

\author{
P. Kadionik *, T. Zimmer ** $\ltimes$, Y. Danto ***. \\ Mis en ligne le 22 février 2002.
}

\begin{abstract}
Résumé
Cet article présente un moyen pédagogique original pour apprendre en ligne à utiliser et maîtriser des équipements de mesure complexes dans le cadre d'une formation universitaire. Ce moyen pédagogique est basé sur les possibilités multimédia inhérentes au réseau Internet « World Wide Web » (WWW) : environnement en réseau, manipulation de documents hypertextes, d'images, de sons...

L'idée de base a été de mettre en oeuvre le concept d'application client/serveur du WWW : on a d'un côté un parc d'instruments de mesure pilotés par une application serveur accessible de n'importe où dans le monde par des applications clientes (ou « browser ») via le réseau Internet. Un système d'identification est de plus mis en oeuvre pour restreindre l'accès au parc d'instruments mis à disposition aux personnes dûment enregistrées. L'application serveur propose en outre le panneau de face avant virtuel de chaque instrument qui pourra être ensuite piloté par le browser de la personne autorisée.

L'intérêt d'une telle approche est ainsi de mettre à disposition de la communauté universitaire un parc d'instruments de mesure performants et récents en vue d'apprendre à les utiliser mais aussi pour réaliser des mesures dans le cadre de recherches via le réseau Internet. On a donc ainsi mis en service sur le « Web» des instruments virtuels $W W W$.

Mots-clés : Microélectronique, Instrumentation, Web, Mesures à distance, Travaux pratiques.
\end{abstract}

(C) EDP Sciences, 2002.

Niveau de connaissances requis. Néant.

Niveau des étudiants. Deuxième cycle : licence ou IUP (EEA ou GEII

\footnotetext{
* Patrice Kadionik

Ingénieur ENSEIRB en 1989.

Docteur de l'Université Bordeaux I en Instrumentation et Mesures en 1992.
}

Patrice Kadionik a travaillé comme Ingénieur Spécialiste Télécom chez SEMA GROUP TELECOM pendant trois ans avent de rejoindre l'ENSEIRB comme Maître de conférences. Rattaché à l'équipe SACT (Synthèse d'Algorithmes et de Circuits pour les Télécommunications) au laboratoire de micoélectronique IXL, il a particulièrement travaillé sur les circuits numériques de compression vidéo. Il collabore depuis peu avec l'équipe Compose du laboratoire d'informatique Labri sur la mise en place d'une plateforme logicielle de développement de services multimédia.

Ses activités d'enseignement concernent l'informatique, l'informatique industrielle et les réseaux.

Adresse postale : ENSEIRB/Université Bordeaux 1, Laboratoire de Microélectronique IXL, 351, cours de la Libération, 34405 Talence Cedex.

email : kadionik@enseirb.fr

http://www.enseirb.fr/ kadionik 
** Thomas Zimmer est maître de conférences à l'Université Bordeaux I où il est en charge de la deuxième année de l'IUP GEII. Dans le cadre de son activité d'enseignant, ses intérêts se concentrent autour de l'instrumentation et la mesure. Il a été notamment le coordinateur du projet européen RETWINE dans le contexte du programme Socrates-Éducation Ouverte et à Distance qui est présenté dans cet article.

Ses activités de recherche se focalisent sur la modélisation électrique de composants, notamment celle du transistor bipolaire à hétérojonction. Dans ce cadre il participe aux programmes consécutifs Européens MEDEA T555 et T204 où il est en charge du work-package « Device development, Modelling and Characterisation ».

a auteur correspondant

Adresse postale : ENSEIRB/Université Bordeaux 1, Laboratoire de Microélectronique IXL, 351, cours de la Libération, 34405 Talence Cedex.

email : zimmer@ixl.u-bordeaux.fr

\begin{abstract}
*** Yves Danto est professeur à l'université de Bordeaux I où il anime le Département de formation EEA, regroupant les cursus Licence-Maîtrise EEA, Licence-Maîtrise SPI, IUP GEII et des DESS.

Il développe ses recherches au laboratoire IXL où il est en charge du groupe Analyse Comportementale et Fiabilité des composants Électroniques.
\end{abstract}

Adresse postale : ENSEIRB/Université Bordeaux 1, Laboratoire de Microélectronique IXL, 351, cours de la Libération, 34405 Talence Cedex.

email : danto@ixl.u-bordeaux.fr

\title{
1. Introduction
}

Le test de composants et systèmes VLSI est fondamental pour le développement des procédés de fabrication, la caractérisation des composants et l'analyse de défaillance. Pour les tests électriques par exemple, on a à disposition une série d'équipements couramment utilisés : système de caractérisation VLSI, analyseur de paramètres électriques, analyseur de réseau, oscilloscope numérique, générateur de fonctions, analyseur de spectre..

La mise en place d'un cours universitaire pratique pour des étudiants en microélectronique concernant l'utilisation et la maîtrise de ces équipements est nécessaire mais n'est pas toujours possible généralement à cause du coût élevé de tels instruments ( $80 \mathrm{KF}$ pour un analyseur de réseau). Ce coût élevé est donc un frein majeur à la mise en place d'un cours universitaire pratique.

L'acquisition d'une telle instrumentation à des fins purement pédagogiques revient donc trop cher et de plus, les équipements seraient dépassés en quelques années.

La durée de vie moyenne d'un cours universitaire pratique est d'environ 10-15 ans, ce qui reste incompatible avec l'évolution rapide de la microélectronique et de son instrumentation.

L'approche originale présentée maintenant est basée sur l'utilisation des concepts multimédia du Web. Cette approche d'enseignement à distance ou télé-enseignement est maintenant possible avec ce formidable moyen de communication qu'est le WWW ; elle va permettre aux étudiants, dans le cadre d'une formation universitaire de maîtriser et d'avoir en plus une expertise dans l'utilisation d'instruments les plus complexes mais aussi les plus récents mis à leur disposition sur le Web.

On appellera par la suite application serveur, une application fournissant un service particulier ; dans notre cas, l'accès et le contrôle d'un instrument virtuel WWW. Par opposition, une application cliente est une application désirant accéder à un service particulier en s'adressant à l'application serveur adéquate. 


\section{Le concept d'instrumentation virtuelle}

Dans chaque laboratoire, il existe toujours un parc d'instruments de mesure qui ne sont pas toujours utilisés à plein temps. Généralement, chaque laboratoire possède ses propres spécificités dans un domaine de recherche particulier : télécommunications, conception d'ASICs, assemblage, conception de circuits VLSI... Le parc d'instruments est ainsi spécifique et donc très ciblé. Mais les étudiants devraient avoir accès à une large palette d'équipements pour posséder une connaissance la plus complète possible. Pour pallier ce problème, nous proposons de mettre en place le concept d'instrument de mesure virtuel accessible par le Web : les instruments et les mesures effectuées sont ainsi géographiquement distincts.

Nous avons d'un côté un parc d'instruments situés n'importe où dans le monde mis à la disposition de la communauté universitaire. Ce parc, composé de la contribution personnelle de chaque laboratoire est par nature des plus complets.

Les instruments de mesure doivent néanmoins posséder une interface GPIB IEEE-488 (General Purpose Interface Bus) pour pouvoir être pilotés par logiciel (interface de contrôle la plus commune dans le domaine de l'instrumentation). Pour un laboratoire donné, les instruments IEEE-488 sont pilotés par une application serveur lancée sur une station de travail connectée au réseau local du laboratoire. Cette application interagira avec le serveur WWW officiel du laboratoire. Les principales fonctions de l'instrument et sa face avant « virtuelle » sont présentées dans une page Web écrite en langage HTML (« Hyper Text Markup Language ») et faisant appel pour la partie graphique à des « applets » écrites en langage Java.

De l'autre côté, nous retrouvons les applications clientes ou browsers Internet qui pourront interagir à distance avec cet instrument via la page Web. L'accès à ce nouveau service Internet d'instrument virtuel ne sera autorisé qu'aux personnes identifiées et reconnues. Le mécanisme d'identification est pris en charge par le serveur WWW du laboratoire pour l'accès réservé à certaines pages Web. Après identification, il est alors possible d'utiliser et de faire des mesures avec l'instrument virtuel.

\section{Implémentation}

La figure 1 présente les équipements nécessaires à la mise en place d'un parc d'instruments virtuels accessibles par le Web. Il est nécessaire d'avoir une station de travail (ou un PC) possédant une carte GPIB pour piloter les instruments IEEE-488.

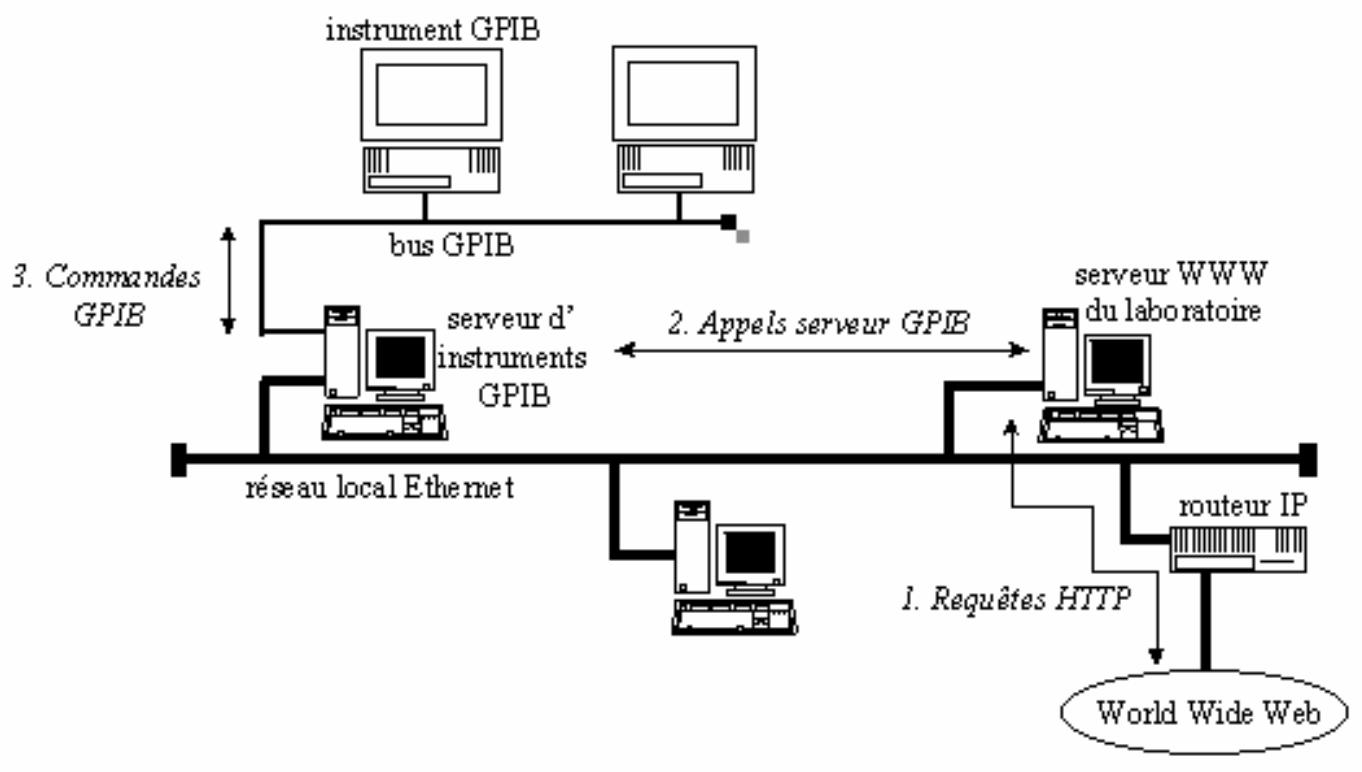

Figure 1. Implémentation d'instruments virtuels WWW. 
Une application serveur doit être développée à cet effet. Cette application peut être par exemple écrite en langage $\mathrm{C}$ dans un environnement UNIX qui possède en standard l'accès aux protocoles Internet. Un serveur WWW (CERN, NCSA ou Apache) doit être aussi mis en place. Il peut être installé sur la station de travail qui doit donc pouvoir accéder au réseau Internet ou mieux sur une deuxième station dédiée pour des raisons d'administration du site et de sécurité. Il convient ensuite de développer les pages HTML où l'on définira les principales fonctions de contrôle de chaque instrument ainsi que sa face avant graphique intégrée sous forme d'une applet écrite en langage Java. L'interaction entre l'application serveur et les requêtes valides reçues par le serveur WWW peut être facilement réalisée à l'aide de requêtes HTML «GET» ou « POST » dans un premier temps mais aussi dans un deuxième temps au niveau de l'applet Java qui offre la possibilité d'exécuter des commandes à distance semblables aux «RPC » Internet (Remote Procedure Call). Ceci oblige donc aussi à écrire des scripts CGI ( (Common Gateway Interface ») pour le contrôle des paramètres de l'utilisateur et pour l'appel de l'application serveur de contrôle des instruments GPIB. Le fait de choisir l'environnement UNIX va permettre de développer très facilement et de façon homogène l'ensemble des composants logiciels nécessaires à la mise en place des instruments virtuels WWW : l'application serveur de contrôle des instruments GPIB sera écrite en langage C; les scripts CGI seront écrits en langage « Shell Script » UNIX ; les applets seront écrites en langage Java et les pages Web en langage HTML.

La figure 2 présente l'interaction entre les différents éléments logiciels mis en oeuvre.

Le mécanisme d'identification d'accès à certaines pages Web sera, enfin, à mettre en place pour identifier par mot de passe l'accès au service d'instrument virtuel. La mise en place d'une base de données indépendante de celle du système d'exploitation UNIX est généralement offerte par le serveur WWW pour la gestion de mots de passe.

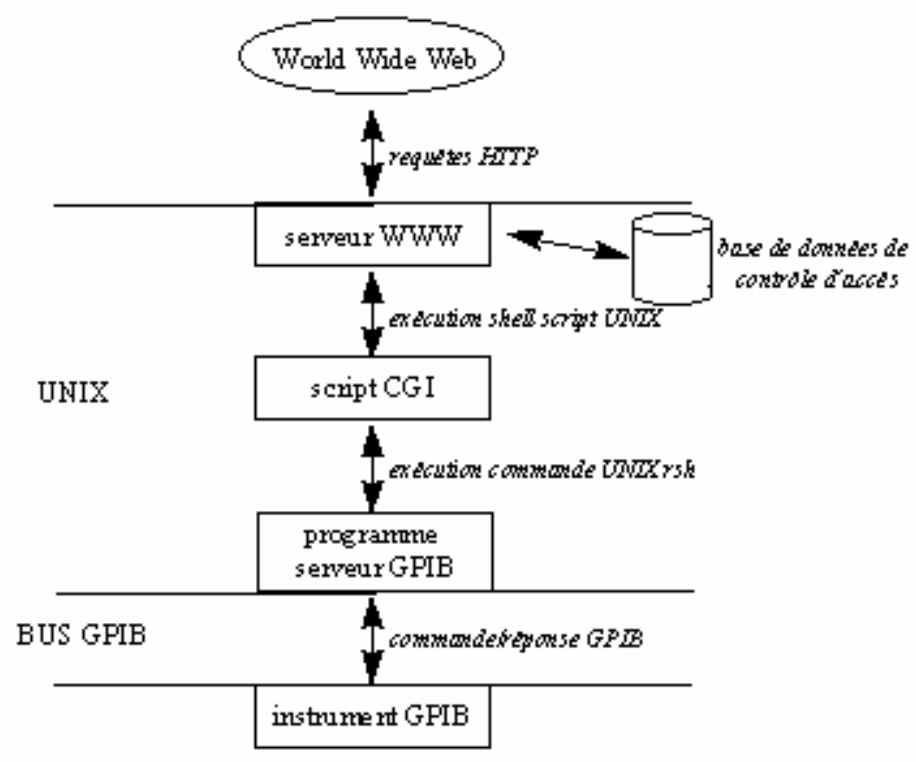

Figure 2. Interaction entre les différents éléments logiciels. 


\section{Exemple d'implémentation}

Nous avons mis en place le système de la figure 1. L'instrument mis à disposition est l'analyseur de paramètres électriques HP 4145. La figure 3 présente sa face avant. L'adresse permettant d'accéder à ce service Internet et en particulier à cet instrument virtuel WWW est : http://www.retwine.net où retwine est l'acronyme de « REmoTe Worldwide Instrument NEtwork ».

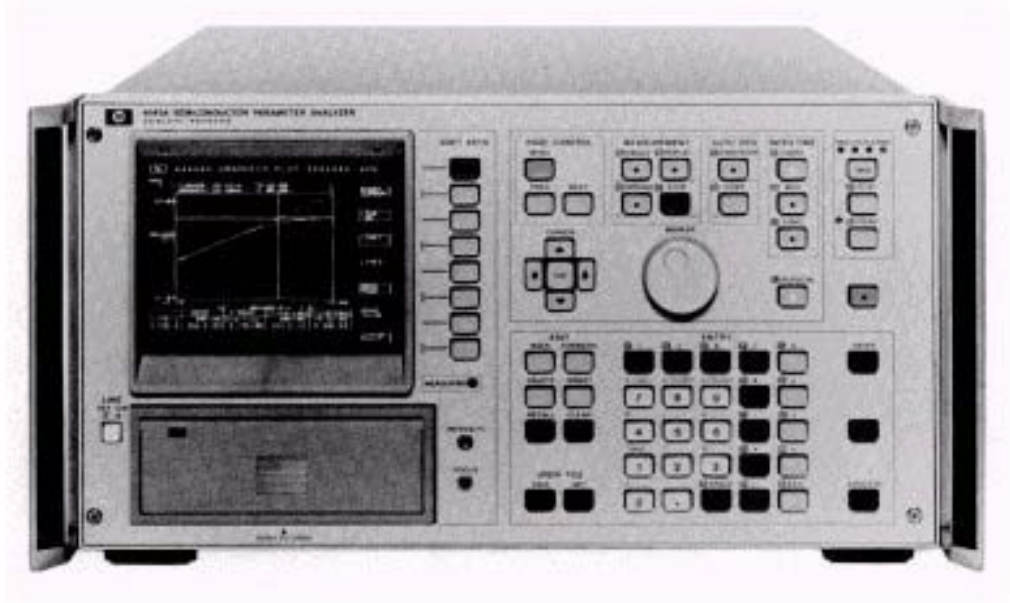

Figure 3. Face avant de l'instrument HP 4145.

Afin de valider l'implémentation proposée d'instruments virtuels WWW, nous avons validé une version de base où l'interaction entre l'instrument et l'utilisateur se fait par l'intermédiaire de formulaires à compléter et où les résultats de mesures sont présentés sous forme de tableaux et résumés dans un fichier récupérable depuis Internet.

\section{Cours pratiques sur le Web}

Ce cours pratique s'adresse essentiellement aux étudiants désirant acquérir une bonne expertise dans l'utilisation d'instruments avancés dédiés à l'électronique. Ce cours comprend une étude détaillée des fonctionnalités, des performances et des limites des mesures réalisées avec cet instrument [1].

Ce cours est divisé en deux parties :

- une première partie concerne la description théorique des fonctionnalités de l'instrument. Les points suivants sont mis en avant : utilisation et champ d'application, principe de la mesure, performances et limitations.

- une deuxième partie concerne l'utilisation pratique de l'instrument. Un premier module permet d'acquérir les bases de maniement de l'appareil et de récupérer les mesures de base. Un deuxième module plus technique propose enfin d'explorer toutes les fonctionnalités avancées de l'instrument.

\section{Discussion}

La mise en place d'un parc d'instruments virtuels accessibles par le Web apporte un ensemble d'avantages dépassant largement le cadre de cet article :

- les étudiants sont toujours en contact avec des instruments nouveaux et performants qui ne peuvent pas être toujours utilisés dans le cadre d'un cours pratique classique. 
- les étudiants des pays en voie de développement peuvent accéder aux instruments les plus modernes.

- la communication entre tous les étudiants est possible en mettant en place un groupe de discussion ou SIG («Special Interest Group ») sous forme d'un groupe d'échange d'informations ( «newsgroup ») accessible depuis Internet.

- le contrôle à distance des instruments virtuels est possible à partir d'autres continents ; ce qui autorise à mettre en place un service de nuit local pour l'accès à l'instrument qui est alors non utilisé.

Nous avons actuellement des laboratoires partenaires en Espagne et en Allemagne qui ont intégré le projet RETWINE et avons mis en place un parc d'instruments virtuels WWW.

\section{Renseignements complémentaires}

Pour tout renseignement complémentaire, vous pouvez contacter retwine@ixl.u-bordeaux.fr.

\section{Références bibliographiques}

[1] T. Zimmer, F. Verdier, Y. Danto, IC Testing Course Performed in Industrial and Research Environment, Proceedings of the European Worshop, Microelectronics Education, Grenoble, France, 5-6 Feb. 1996. 